\title{
On the Collisional Damping of Giant Dipole Resonance
}

\author{
Osman Yilmaz ${ }^{1}$, Ahmet Gokalp ${ }^{1}$, Serbulent Yildirim ${ }^{1}$ and Sakir Ayik ${ }^{2}$ \\ 1 Physics Department, Middle East Technical University, \\ 06531 Ankara, Turkey \\ ${ }^{2}$ Physics Department, Tennessee Technological University, Cookeville TN 38505, USA
}

(September 2, 2018)

\begin{abstract}
Collisional damping widths of giant dipole excitations are calculated in Thomas-Fermi approximation by employing the microscopic in-medium crosssections of $\mathrm{Li}$ and Machleidt and the phenomenological Gogny force. The results obtained in both calculations compare well, but account for about $25-35 \%$ of the observed widths in ${ }^{120} \mathrm{Sn}$ and ${ }^{208} \mathrm{~Pb}$ at finite temperatures. 21.30.Fe, $24.30 . \mathrm{Cz}$
\end{abstract}

Typeset using REVTEX 
During last several years, much work has been done to understand the damping properties of giant resonance excitations at zero and finite temperature [1,2]. In medium-weight and heavy nuclei at relatively low temperatures the overwhelming contribution to damping arises from the spreading width $\Gamma^{\downarrow}$ due to mixing of collective excitations with more complicated doorway states. There are essentially two different approaches for calculation of the spreading widths: (i) Coherent mechanism due to coupling with low-lying surface modes which provides an important mechanism for damping of giant resonance in particular at low temperatures [3 6], (ii) Damping due to the coupling with incoherent 2p-2h states which is usually referred to as the collisional damping [7] 10], and the Landau damping modified by two-body collisions [11-13]. Calculations carried out on the basis of these approaches are partially successful in explaining the broadening of the giant dipole resonance (GDR) with increasing excitation energy. In particular, the model based on the coupling with thermal shape fluctuations in adiabatic approximation provides a reasonable description for the dipole strength distribution in ${ }^{208} \mathrm{~Pb}$ and the ${ }^{120} \mathrm{Sn}$ nuclei [14 [16]. In this work, we do not consider the coherent contribution to damping, but investigate the collisional damping at finite temperature due to decay of the collective state into incoherent $2 \mathrm{p}-2 \mathrm{~h}$ excitations in the basis of a non-Markovian transport approach. In order to assess how much of the total width of giant resonance excitations is exhausted by decay into the incoherent $2 \mathrm{p}-2 \mathrm{~h}$ states, we need realistic in-medium cross-sections which interpolate correctly between the free space and the medium. In previous investigations, incoherent contributions have been estimated by employing either free nucleon-nucleon cross sections or an effective Skyrme force, which provide, at most, a semi-quantitative description of the collisional damping widths [17]. In this respect, the Skyrme force provides a poor approximation in the collision term, since in the vicinity of nuclear surface, it does not match at all to the free nucleon-nucleon crosssections. In this letter, we present calculations performed in Thomas-Fermi approximation by employing the microscopic in-medium cross-sections calculated by Li and Machleidt [18], which interpolate correctly between the free space and the medium, and provide the best available input for determining the magnitude of the collisional damping at finite tempera- 
ture. For comparison, we also carry out calculations using the phenomenological finite range Gogny force and the zero range Skyrme force [19,20].

The small amplitude limit of the extended TDHF provides an appropriate basis for investigating collective vibrations, in which damping due the incoherent $2 \mathrm{p}-2 \mathrm{~h}$ decay is included in the form of a non-Markovian collision term [21]. Then, a description for small density fluctuations, $\delta \rho(t)=\rho(t)-\rho_{0}$, is obtained by linearizing the extended TDHF theory around a finite temperature equilibrium state $\rho_{0}$ [8,22],

$$
\begin{aligned}
& i \hbar \frac{\partial}{\partial t} \delta \rho-\left[h_{0}, \delta \rho\right]-\left[\delta U, \rho_{0}\right]= \\
& -\frac{i}{\hbar} \int^{t} d t^{\prime} \operatorname{Tr}_{2}\left[v, \rho_{1}^{0} \rho_{2}^{0} e^{-i h_{0}\left(t-t^{\prime}\right)}\left[Q\left(t^{\prime}\right), v\right] e^{+i h_{0}\left(t-t^{\prime}\right)}\left(1-\rho_{1}^{0}\right)\left(1-\rho_{2}^{0}\right)\right]-\text { h.c. }
\end{aligned}
$$

where $\delta U$ represents small deviations in the effective mean-field potential. The right hand side describes a non-Markovian quantal collision term in which the quantity $Q(t)$ denotes the distortion matrix associated with the single particle density matrix, $\delta \rho(t) \equiv\left[Q(t), \rho_{0}\right]$. It is convenient to analyze collective vibrations by expanding the small deviation $\delta \rho(t)$ in terms of finite temperature RPA modes $O_{\lambda}^{\dagger}$ and $O_{\lambda}$,

$$
\delta \rho(t)=\sum_{\lambda>0} z_{\lambda}(t)\left[O_{\lambda}^{\dagger}, \rho_{0}\right]-z_{\lambda}^{*}(t)\left[O_{\lambda}, \rho_{0}\right]
$$

where $z_{\lambda}(t)$ and $z_{\lambda}^{*}(t)$ denote the amplitudes associated with the RPA modes, $O_{\lambda}^{\dagger}$ and $O_{\lambda}$, which are determined by the finite temperature RPA equation [23,24. Substituting this expansion into eq.(1) and projecting by $O_{\lambda}$, and noting that $Q(t)=\sum z_{\lambda}(t) O_{\lambda}^{\dagger}-z_{\lambda}^{*}(t) O_{\lambda}$, we find that the amplitudes of the RPA modes execute damped harmonic motion [8,17]

$$
i \hbar \frac{d}{d t} z_{\lambda}-\hbar \omega_{\lambda} z_{\lambda}=\int^{t} d t^{\prime} \Sigma_{\lambda}\left(t-t^{\prime}\right) z_{\lambda}\left(t^{\prime}\right)
$$

where the right hand side describes the self-energy of the collective mode due to coupling to $2 \mathrm{p}-2 \mathrm{~h}$ excitations. In the Hartree-Fock representation, the Fourier transform of the selfenergy is given by,

$$
\Sigma_{\lambda}(\omega)=\frac{1}{4} \sum \frac{|<i j|\left[O_{\lambda}^{\dagger}, v\right]\left|k l>_{A}\right|^{2}}{\hbar \omega-\Delta \epsilon+i \eta}\left[n_{k} n_{l} \bar{n}_{i} \bar{n}_{j}-n_{i} n_{j} \bar{n}_{k} \bar{n}_{l}\right]
$$


where $\bar{n}_{i}=1-n_{i}, \Delta \epsilon=\epsilon_{i}+\epsilon_{j}-\epsilon_{k}-\epsilon_{l}$, and $\eta$ is a small positive number. The self-energy agrees with the expression written down by Landau [25], in which the real and imaginary parts, $\Sigma_{\lambda}(\omega)=\Delta_{\lambda}(\omega)-\frac{i}{2} \Gamma_{\lambda}(\omega)$, determine the energy shift and the damping width of the collective excitation, respectively [4].

We consider the expression for the self-energy in the Thomas-Fermi approximation, which corresponds to a semi-classical transport description of the collective vibrations. It can be shown that the $2 \mathrm{p}-2 \mathrm{~h}$ self-energy in Thomas-Fermi approximation can be deduced from the quantal expression by replacing the occupation numbers with the equilibrium phase-space density given by the Fermi-Dirac function $n_{i} \rightarrow f(\epsilon, T)=1 /[\exp (\epsilon-\mu) / T+1]$ with $\mu$ as the chemical potential, and summations over the $2 \mathrm{p}-2 \mathrm{~h}$ states with integrals over phase-space, $\Sigma \rightarrow \int d^{3} r d^{3} p_{1} d^{3} p_{2} d^{3} p_{3} d^{3} p_{4}$ [8.26]. Furthermore, spin-isospin effects can be included into the treatment by considering proton and neutron degrees of freedom separately. The small deviations of the density matrices $\delta \rho_{p}(t), \delta \rho_{n}(t)$ of protons and neutrons are determined by two coupled equations analogous to eq.(1). The collision terms in these equations involve binary collisions between proton-proton, neutron-neutron and proton-neutron, and a summation over the spins of the colliding particles. Since in isoscalar/isovector modes, proton and neutron densities vibrate in-phase/out-of phase, $\delta \rho_{p}(t)=\mp \delta \rho_{n}(t)$, we can deduce equation of motions for describing isoscalar/isovector vibrations by adding and subtracting the corresponding equations for protons and neutrons. As a result, in Thomas-Fermi approximation, we obtain for the collisional widths of isovector modes [8],

$$
\Gamma_{\lambda}^{v}=\frac{1}{N_{\lambda}} \int d \mathbf{p}_{1} d \mathbf{p}_{2} d \mathbf{p}_{3} d \mathbf{p}_{4}\left[\left(W_{p p}+W_{n n}\right)\left(\frac{\Delta \chi_{\lambda}}{2}\right)^{2}+2 W_{p n}\left(\frac{\widetilde{\Delta \chi_{\lambda}}}{2}\right)^{2}\right] Z f_{1} f_{2} \bar{f}_{3} \bar{f}_{4}
$$

and a similar expression for the isoscalar modes. Here, $N_{\lambda}=\int d \mathbf{r} d \mathbf{p}\left(\chi_{\lambda}\right)^{2}\left(-\frac{\partial}{\partial \epsilon} f\right)$ is a normalization, $\Delta \chi_{\lambda}=\chi_{\lambda}(1)+\chi_{\lambda}(2)-\chi_{\lambda}(3)-\chi_{\lambda}(4), \widetilde{\Delta \chi_{\lambda}}=\chi_{\lambda}(1)-\chi_{\lambda}(2)-\chi_{\lambda}(3)+\chi_{\lambda}(4)$, $Z=\left[\delta\left(\hbar \omega_{\lambda}-\Delta \epsilon\right)-\delta\left(\hbar \omega_{\lambda}+\Delta \epsilon\right)\right] / \hbar \omega_{\lambda}$, and $\chi_{\lambda}(t)$ denotes the distortion factor of the phase-space density $\delta f(t)=\chi_{\lambda}(t)(-\partial f / \partial \epsilon)$ in the corresponding mode. In this expression, transition rates $W_{p p}, W_{n n}, W_{p n}$ associated with proton-proton, neutron-neutron and protonneutron collisions are given in terms of the corresponding scattering cross-sections as, 


$$
W(12 ; 34)=\frac{1}{(2 \pi \hbar)^{3}} \frac{4 \hbar}{m^{2}} \frac{d \sigma}{d \Omega} \delta\left(\mathbf{p}_{1}+\mathbf{p}_{2}-\mathbf{p}_{3}-\mathbf{p}_{4}\right)
$$

We apply the formula (5) to calculate the collisional widths of the GDR excitations by taking distortion factors of the momentum distribution according to the scaling picture as $\chi_{D}(\mathbf{p})=\mathbf{p} \cdot \mathbf{e}$, where $\mathbf{e}$ denotes the unit vector along the relative displacement of the proton and neutron momentum distributions. Due to momentum conservation, terms involving $W_{p p}$ and $W_{n n}$ drop out, and the damping is determined by the proton-neutron collision term. The spin averaged proton-neutron cross-section associated with an effective residual interaction can be expressed as,

$$
\left(\frac{d \sigma}{d \Omega}\right)_{p n}=\frac{\pi}{(2 \pi \hbar)^{3}} \frac{m^{2}}{4 \hbar} \cdot \frac{1}{8} \sum_{S, T}(2 S+1)|<\mathbf{q} ; S, T| v\left|\mathbf{q}^{\prime} ; S, T>_{A}\right|^{2}
$$

where $\mathbf{q}=\left(\mathbf{p}_{1}-\mathbf{p}_{2}\right) / 2, \mathbf{q}^{\prime}=\left(\mathbf{p}_{3}-\mathbf{p}_{4}\right) / 2$ are the relative momenta before and after a binary collision, and $<\mathbf{q} ; S, T|v| \mathbf{q}^{\prime} ; S, T>_{A}$ represents the fully anti-symmetric matrix element of the residual interaction between two particle states with total spin and isospin $S$ and T. By noting that, $\mathrm{S}=\mathrm{T}=1$ and $\mathrm{S}=\mathrm{T}=0$ matrix elements of the interaction are space antisymmetric, and $\mathrm{S}=1, \mathrm{~T}=0$ and $\mathrm{S}=0, \mathrm{~T}=1$ matrix elements are space symmetric, we find that proton-neutron cross-section associated with the Gogny force is given,

$$
\begin{aligned}
\left(\frac{d \sigma}{d \Omega}\right)_{p n}^{G}= & \frac{\pi}{(2 \pi \hbar)^{3}} \frac{m_{G}^{*}}{4 \hbar} \frac{1}{8}\left\{3\left|\sum_{i=1}^{2} I_{i}^{-}\left(W_{i}+B_{i}-H_{i}-M_{i}\right)\right|^{2}+\left|\sum_{i=1}^{2} I_{i}^{-}\left(W_{i}-B_{i}+H_{i}-M_{i}\right)\right|^{2}+\right. \\
& \left.3\left|\sum_{i=1}^{2} I_{i}^{+}\left(W_{i}+B_{i}+H_{i}+M_{i}\right)+4 t_{3} \rho^{1 / 3}\right|^{2}+\left|\sum_{i=1}^{2} I_{i}^{+}\left(W_{i}-B_{i}-H_{i}+M_{i}\right)\right|^{2}\right\}
\end{aligned}
$$

where $m_{G}^{*}$ denotes the effective mass corresponding to the Gogny force, and the quantities $I_{i}^{+}$and $I_{i}^{-}$are the symmetric and anti-symmetric matrix elements of the Gaussian factor in the force,

$$
I_{i}^{ \pm}=\left(\sqrt{\pi} \mu_{i}\right)^{3}\left(\exp \left[-\frac{1}{4}\left(\mathbf{q}-\mathbf{q}^{\prime}\right)^{2}\left(\frac{\mu_{i}}{\hbar}\right)^{2}\right] \pm \exp \left[-\frac{1}{4}\left(\mathbf{q}+\mathbf{q}^{\prime}\right)^{2}\left(\frac{\mu_{i}}{\hbar}\right)^{2}\right]\right)
$$

In these expressions, $\rho$ is the local density and $W_{i}, B_{i}, H_{i}, M_{i}, \mu_{i}$ denote the standart parameters of the Gogny force [19:20]. In a similar manner, we can calculate the cross-section associated with the Skyrme force and obtain [8], 


$$
\begin{gathered}
\left(\frac{d \sigma}{d \Omega}\right)_{p n}^{S}=\frac{\pi}{(2 \pi \hbar)^{3}} \frac{m_{S}^{* 2}}{4 \hbar} \frac{1}{2}\left(\left[t_{0}\left(1-x_{0}\right)+\frac{t_{1}}{2 \hbar^{2}}\left(1-x_{1}\right)\left(\mathbf{q}^{2}+\mathbf{q}^{\prime 2}\right)+\frac{t_{3}}{6}\left(1-x_{3}\right) \rho^{\alpha}\right]^{2}+\right. \\
3\left[t_{0}\left(1+x_{0}\right)+\frac{t_{1}}{2 \hbar^{2}}\left(1+x_{1}\right)\left(\mathbf{q}^{2}+\mathbf{q}^{\prime 2}\right)+\frac{t_{3}}{6}\left(1+x_{3}\right) \rho^{\alpha}\right]^{2}+ \\
\left.\left[\left(\frac{t_{2}}{\hbar^{2}}\right)^{2}\left(1-x_{2}\right)^{2}+3\left(\frac{t_{2}}{\hbar^{2}}\right)^{2}\left(1+x_{2}\right)^{2}\right]\left(\mathbf{q} \cdot \mathbf{q}^{\prime}\right)^{2}\right)
\end{gathered}
$$

The expressions for the effective masses $m_{G}^{*}(r)$ and $m_{S}^{*}(r)$ for the Gogny and Skyrme forces can be found in [19,20]. The angle $\Theta$ between $\mathbf{q}$ and $\mathbf{q}^{\prime}$ in the cross-sections (9) and (13) defines the scattering angle in the center of mass frame, and the total cross-section is found by an integration over this angle, $\sigma_{p n}=2 \pi \int \sin \Theta d \Theta(d \sigma / d \Omega)_{p n}$. This integral is trivial for the Skyrme cross-section, but a numerical integration is needed over the scattering angle to find the total proton-neutron cross-section for the Gogny force.

In figures 1 and 2, we compare the microscopic in-medium cross-sections of Li and Machleidt with the cross-sections (dotted lines) of the Gogny force (dashed lines) and the Skyrme force (solid lines) with the $\mathrm{SkM}^{*}$ parameters. For this purpose we employ a convenient parameterization of the microscopic calculations as reported in [18],

$$
\sigma_{p n}^{L M}=\left[31.5+0.092\left|20.2-E_{l a b}^{0.53}\right|^{2.9}\right] \frac{1.0+0.0034 E_{l a b}^{1.51} \rho^{2}}{1.0+21.55 \rho^{1.34}}
$$

where $E_{l a b}=\left(\mathbf{p}_{1}-\mathbf{p}_{2}\right)^{2} / 2 m=2 \mathbf{q}^{2} / m$ is the kinetic energy of the projectile in the rest frame of the target nucleon which is also equal to twice the energy available in the center of mass frame. Figure 1 illustrates the total cross-sections as a function of the bombarding energy $E_{l a b}$ at two different nuclear matter densities $\rho=\rho_{0}=0.18 \mathrm{fm}^{-3}$ (top panel) and $\rho=\rho_{0} / 2$ (bottom panel). The cross-sections shown in the left and right parts of the figure are calculated with the bare nucleon mass and the corresponding effective masses, respectively. Around the normal matter density $\rho \approx \rho_{0}$, and over a narrow energy interval around $150 \mathrm{MeV}$, these cross-sections roughly match, however the phenomenological crosssections deviate strongly from the microscopic cross-sections at lower densities and lower and higher energies. Discrepancies are even larger in the calculations with the bare nucleon mass. In figure 2, the cross-sections are plotted as a function of density at the bombarding energy $E_{l a b}=100 \mathrm{MeV}$. For decreasing density, the microscopic calculations approach the 
free proton-neutron cross-section and compare well with the experimental data, where as the phenomenological cross-sections strongly increase and reach large values in free space. From these observations, we can safely state that the microscopic calculations of Li and Macleidt provide a more reliable description of the in-medium cross-sections than those given by the Gogny and Skyrme type forces.

In a previous work, the momentum integrals in the expression (5) for the damping width are evaluated in an approximate manner using a method familiar from the Fermi liquid theory. The method subsequently improved by incorporating the surface corrections [8]. Here, we perform the momentum integrals exactly. For this purpose it is convenient to transform the variable into the relative momentum and total momentum before and after the collision, $\mathbf{q}=\left(\mathbf{p}_{1}-\mathbf{p}_{2}\right) / 2, \mathbf{P}=\mathbf{p}_{1}+\mathbf{p}_{2}$, and $\mathbf{q}^{\prime}=\left(\mathbf{p}_{3}-\mathbf{p}_{4}\right) / 2, \mathbf{P}^{\prime}=\mathbf{p}_{3}+\mathbf{p}_{4}$. Then, in expression (5), the integral over $\mathbf{P}^{\prime}$ is done immediately to give

$$
\Gamma_{D}=\frac{2}{N_{D}} \int d^{3} r\left[\frac{1}{(2 \pi \hbar)^{3}} \frac{4 \hbar}{m^{* 2}}\right] \frac{1}{\hbar \omega_{D}}\left[1-\exp \left(-\frac{\hbar \omega_{D}}{T}\right)\right] I_{D}(r)
$$

with

$$
I_{D}(r)=\int d^{3} P d^{3} q d^{3} q^{\prime}\left(\mathbf{q} \cdot \mathbf{e}-\mathbf{q}^{\prime} \cdot \mathbf{e}\right)^{2}\left(\frac{d \sigma}{d \Omega}\right)_{p n} \delta\left(\hbar \omega_{D}-\epsilon^{\prime}+\epsilon\right) f_{1} f_{2} \bar{f}_{3} \bar{f}_{4}
$$

where $\epsilon=\mathbf{q}^{2} / m^{*}$ and $\epsilon^{\prime}=\mathbf{q}^{\prime 2} / m^{*}$ represent energies of two particle system in the center of mass frame before and after the collision. In terms of the integration variables, the products of the phase-space factors have the following forms,

$$
f_{1} f_{2}=\frac{1}{1+A^{2}(E, \epsilon)+A(E, \epsilon)(\exp (+z \sqrt{E \epsilon} / T)+\exp (-z \sqrt{E \epsilon}) / T)}
$$

and

$$
\bar{f}_{3} \bar{f}_{4}=\frac{A^{2}\left(E, \epsilon^{\prime}\right)}{1+A^{2}\left(E, \epsilon^{\prime}\right)+A\left(E, \epsilon^{\prime}\right)\left(\exp \left(+z^{\prime} \sqrt{E \epsilon^{\prime}}\right) / T+\exp \left(-z^{\prime} \sqrt{E \epsilon^{\prime}}\right) / T\right)}
$$

where $A(E, \epsilon)=\exp \left((E+\epsilon) / 2-\epsilon_{F}\right) / T$, and $z=\cos \theta$ and $z^{\prime}=\cos \theta^{\prime}$ are the angles between $\mathbf{q}$ and $\mathbf{P}$, and $\mathbf{q}^{\prime}$ and $\mathbf{P}$, respectively. In the further evaluation of the momentum integrals, we neglect the angular anisotropy of the cross-sections and make the replacement $(d \sigma / d \Omega)_{p n} \rightarrow \sigma_{p n} / 4 \pi$ to obtain, 


$$
I_{D}(r)=(2 \pi)^{2}\left(m^{*} \sqrt{m^{*}}\right)^{3} \frac{m^{*}}{3} \int_{0}^{\infty} \int_{0}^{\infty} \sqrt{E} d E \sqrt{\epsilon \epsilon^{\prime}} d \epsilon \sigma_{p n}\left(\epsilon+\epsilon^{\prime}\right) \int_{-1}^{+1} \int_{-1}^{+1} d z d z^{\prime} f_{1} f_{2} \bar{f}_{3} \bar{f}_{4}
$$

where $\epsilon^{\prime}=\epsilon+\hbar \omega_{D}$ and $E=P^{2} / 4 m^{*}$ denotes the kinetic energy of the center of mass. In obtaining this result, we make the replacement $\left(\mathbf{q} \cdot \mathbf{e}-\mathbf{q}^{\prime} \cdot \mathbf{e}\right)^{2} \rightarrow\left(\mathbf{q}^{2}+\mathbf{q}^{\prime 2}\right) / 3$ in the integrand of (13), which follow from the fact that the integral of the cross terms vanishes and the integral is independent of the orientation of the unit vector $\mathbf{e}$. It is possible to carry out the angular integrals over $z$ and $z^{\prime}$ analytically, and we perform the remaining three fold integrations over $E, \epsilon$ and the radial coordinate $r$ numerically.

In the numerical evaluations, we determine the nuclear density $\rho(r)$ in Thomas-Fermi approximation using a Wood-Saxon potential with a depth $V_{0}=-44 \mathrm{MeV}$, thickness $a=0.67$ fm and sharp radius $R_{0}=1.27 A^{1 / 3} \mathrm{fm}$, calculate the position dependent chemical potential $\mu(r, T)$ in the Fermi-Dirac function $f(\epsilon, T)$ at each temperature, and use the formula $\hbar \omega=80 A^{-1 / 3} \mathrm{MeV}$ for the GDR energies. In Fermi gas picture, the effective excitation energy of the system and temperature are related according to,

$$
E^{*}=\int \frac{4}{(2 \pi \hbar)^{3}} d^{3} r d^{3} p \epsilon[f(\epsilon, T)-f(\epsilon, T=0)]
$$

For temperatures small compared to the Fermi energy $\epsilon_{F}=\mu(T=0)$ and in sharp radius approximation, the relation between the excitation energy and temperature becomes, $E^{*}=$ $a_{F} T^{2}$, where $a_{F}=A \pi^{2} / 4 \epsilon_{F}$ is the Fermi gas level density parameter. The experimental temperature $T^{*}$ is determined from the excitation energy using a similar relation $T^{*}=$ $\sqrt{E^{*} / a_{E}}$, where $a_{E}$ denotes the energy dependent empirical level density parameter [2]. Hence, the temperature parameter in $f(\epsilon, T)$ is related to the experimental temperature as $T=T^{*} \sqrt{a_{E} / a_{F}}$. Figure 3 shows the collisional damping width of GDR in ${ }^{120} \mathrm{Sn}$ and ${ }^{208} \mathrm{~Pb}$ as a function of the experimental temperature and comparison with data. Calculations performed with the cross-sections of Li and Machleidt are indicated with dotted lines. For comparison, we also indicate the results with the $\mathrm{SkM}^{*}$ (solid lines) and the Gogny (dashed lines) cross-sections with the bare nucleon mass (lower panel) and the effective nucleon mass (upper panel), and the points are the experimental data [2]. The calculations with 
cross-sections of Li and Machleidt exhibit a weaker temperature dependence than data and account for about $25-35 \%$ of the experimental damping widths in tin and lead nuclei at finite temperatures. As discussed above, the calculations with Gogny and Skyrme forces are not reliable, since the phenomenological cross-sections do not interpolate correctly between the free space and the medium. Their magnitude at low densities and in the vicinity of Fermi energy, where the dominant contributions to damping arises, become much larger than the cross-sections of Li and Machleidt. As a result, the phenomenological forces predict larger damping, although, the magnitude of the damping is reduced by the effective mass, as seen from the top panel of figure 3. However, it is surprising that, the damping width calculated with the Gogny force including the effective mass reduction almost agree with the results obtained with the cross-sections of the Li and Macleidt for both tin and lead nuclei. Figure 4 illustrates a comparison of the calculations with the revised data taken from [16]. In the revised data, the effective temperatures are smaller and the damping widths show a stronger temperature dependence than reported in [2]. Consequently, the collisional damping accounts for a smaller fraction of the experimental damping widths.

In order to assess the fraction of the total width of collective excitations that is exhausted by decay into the incoherent $2 \mathrm{p}-2 \mathrm{~h}$ states, namely the collisional damping, we need realistic in-medium nucleon-nucleon cross-sections around Fermi energy. In this respect, microscopic in-medium cross-sections of $\mathrm{Li}$ and Machleidt provides the best available input, since the cross-sections interpolate correctly between the free space and the medium. In contrast, the cross-sections based on the phenomenological Skyrme force and even the finite range Gogny force exhibit unrealistic behavior as a function of density and energy. In the present investigations, by employing cross-sections of Li and Machleidt, we carry out calculations of the damping widths of giant dipole excitations in Thomas-Fermi approximation at finite temperature, and compare the results with the GDR measurements in ${ }^{120} \mathrm{Sn}$ and ${ }^{208} \mathrm{~Pb}$ nuclei. We also compare the results with calculations based on the Skyrme and Gogny cross-sections. To the extend that we accept the validity of the in-medium cross-sections of Li and Machleidt, we can conclude that the collisional damping of the GDR excitations is not very strong, and 
accounts for about $1 / 3$ of $\Gamma^{\downarrow}$ in both nuclei at zero and finite temperatures. Consequently, there is plenty of room remains for coherent damping mechanism due to coupling of the dipole mode with surface fluctuations.

\section{Acknowledgments}

One of us (S. A.) gratefully acknowledges the Physics Department of Middle East Technical University for warm hospitality extended to him during his visits. We thank D. Kusnezov for providing the revised data of ${ }^{120} \mathrm{Sn}$ and ${ }^{208} \mathrm{~Pb}$, R. Machleidt for providing a table of their cross-sections, and P. Schuck for fruitful discussions. This work is supported in part by the U.S. DOE Grant No. DE-FG05-89ER40530. 


\section{REFERENCES}

[1] H. J. Hofmann et al., Nucl. Phys. A571 (1994) 301.

[2] T. Baumann et al., Nucl. Phys. A635(1998)428.

[3] G. F. Bertsch, P. F. Bortignon and R. A. Broglia, Rev. of Mod. Phys. 55 (1983) 287.

[4] J. Wambach, Prog. Phys. 51 (1988) 989.

[5] B. Lauritzen, P. F. Bortignon, R. A. Broglia and V. G. Zelevinsky, Phys. Rev. Lett. 74 (1995) 5190.

[6] P. Chomaz, Ann. Phys. Fr. 21(1996)669; 55(1983)287.

[7] M. Belkacem, S. Ayik and A. Bonasera, Phys. Rev. C52 (1995) 2499.

[8] S. Ayik, O. Yilmaz, A. Gokalp and P. Schuck, Phys. Rev. C58 (1998) 1594.

[9] U. Furmann, K. Morawetz and R. Welke, Phys. Rev. C58 (1998) 1473.

[10] D. Lacroix, P. Chomaz and S. Ayik, Phys. Rev. C58 (1998) 2154.

[11] A. Smerzi, A. Bonasera and M. diToro, Phys. Rev. C44 (1991) 1713.

[12] V. Baran et al., Nucl. Phys. A599 (1996) 29c.

[13] V. M. Kolomietz, V. A. Plujko and S. Shlomo, Phys. Rev. C54 (1996) 3014.

[14] W.E. Ormand, P. F. Bortignon and R. A. Broglia, Phys. Rev. Lett. 77 (1996) 607.

[15] W.E. Ormand, P. F. Bortignon, R. A. Broglia and A. Bracco, Nucl. Phys. A614 (1997) 217.

[16] D. Kusnezov, Y. Alhassid and K. A. Snover, Phys. Rev. Lett. 81 (1998) 542.

[17] S. Ayik, D. Lacroix and P. Chomaz, preprint GANIL P 99 23, and submitted to Nucl. Phys. A.

[18] M. G. Q. Li and R. Machleidt, Phys. Rev. C48 (1993) 1702; C49 (1994) 566. 
[19] P. Schuck et al., Prog. Part. Nucl. Phys. Vol. 22 (1989) 181.

[20] P. Ring and P. Schuck, The Nuclear Many Body Problem, Springer, Berlin (1980).

[21] Y. Abe, S. Ayik, P. G. Reinhard and E. Suraud, Phys. Rep. 275 (1996) 49.

[22] S. Ayik, D. Lacroix and P. Chomaz, Advances in Nuclear Dynamics, 5, ed. W. Bauer (Plenum, New York, 1999).

[23] D. Vautherin and N. Vinh Mau, Nucl. Phys. A422 (1984) 140.

[24] H. Sagawa and G. F. Bertsch, Phys. Lett. B146(1984) 138.

[25] L. D. Landau, Sov. Phys. JETP 5 (1957) 101.

[26] R. Hasse, in Proceedings of Phase Space Approach to Nuclear Dynamics, eds. M. diToro, W. Norenberg, M. Rosina and S. Stringari (World Scientific, Singapore, 1985). 


\section{Figure Captions:}

Figure 1: The proton-neutron in-medium cross-sections as a function of bombarding energy $E_{l a b}$ at several different densities. Dotted lines are cross-sections of Li and Machleidt, and solid and dashed lines are cross-sections associated with the $\mathrm{SkM}^{*}$ and the Gogny forces with the bare nucleon mass (left) and the effective nucleon mass (right), respectively.

Figure 2: The proton-neutron in-medium cross-sections as a function of density $\rho$ at $E_{l a b}=$ $100 \mathrm{MeV}$. Dotted lines are cross-sections of Li and Machleidt, and solid and dashed lines are cross-sections with the $\mathrm{SkM}^{*}$ and the Gogny forces with the bare nucleon mass (left) and the effective nucleon mass (right), respectively.

Figure 3: The collisional damping width of GDR in ${ }^{120} \mathrm{Sn}$ and ${ }^{208} \mathrm{~Pb}$ as a function of temperature. Dotted lines are calculations with the cross-sections of Li and Machleidt, and solid and dashed lines are results with the SkM* and the Gogny cross-sections with the bare nucleon mass (lower panel) and the effective nucleon mass (upper panel). The data is taken from [2].

Figure 4: Same as in figure 3, but shown with the revised data taken from 16. 


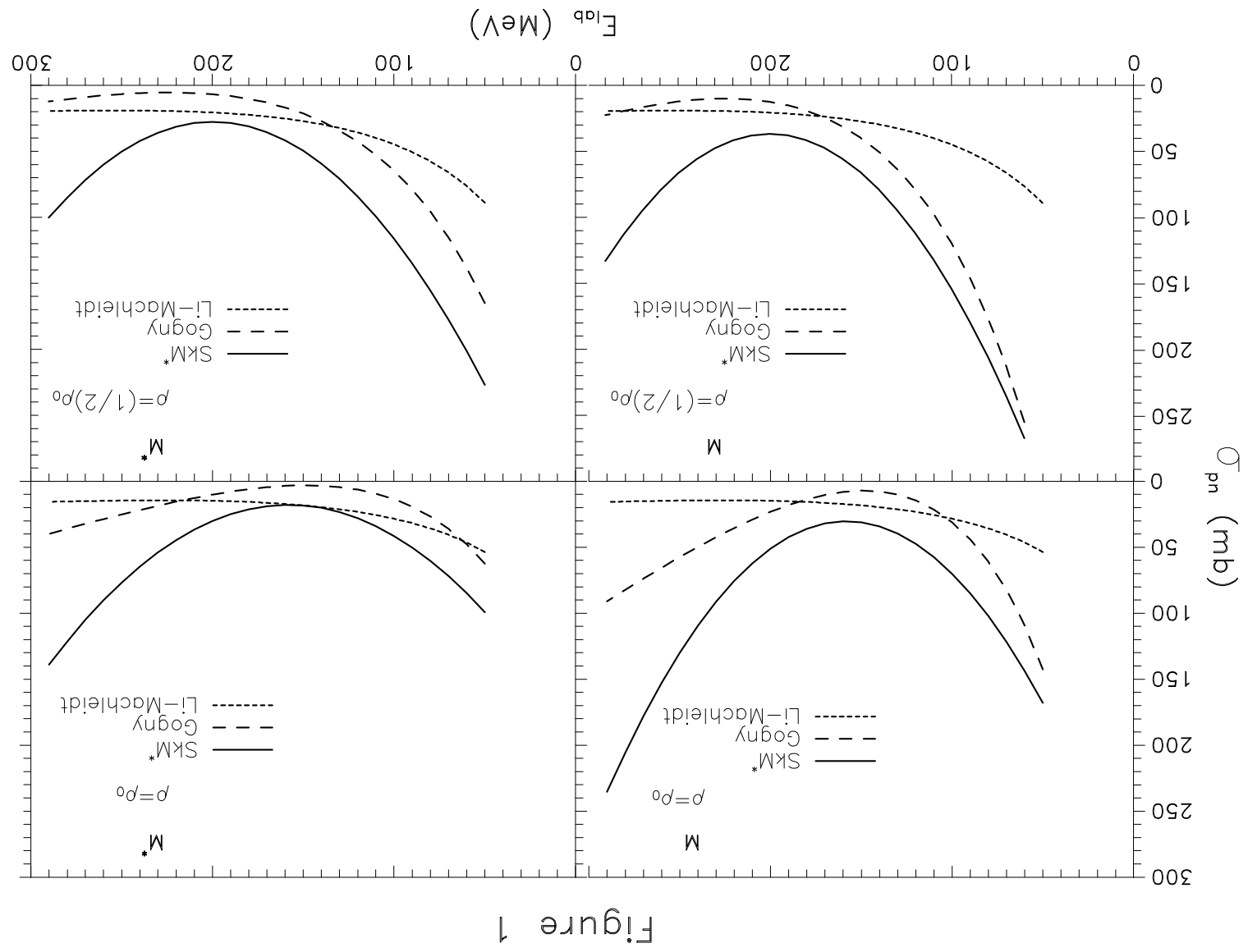




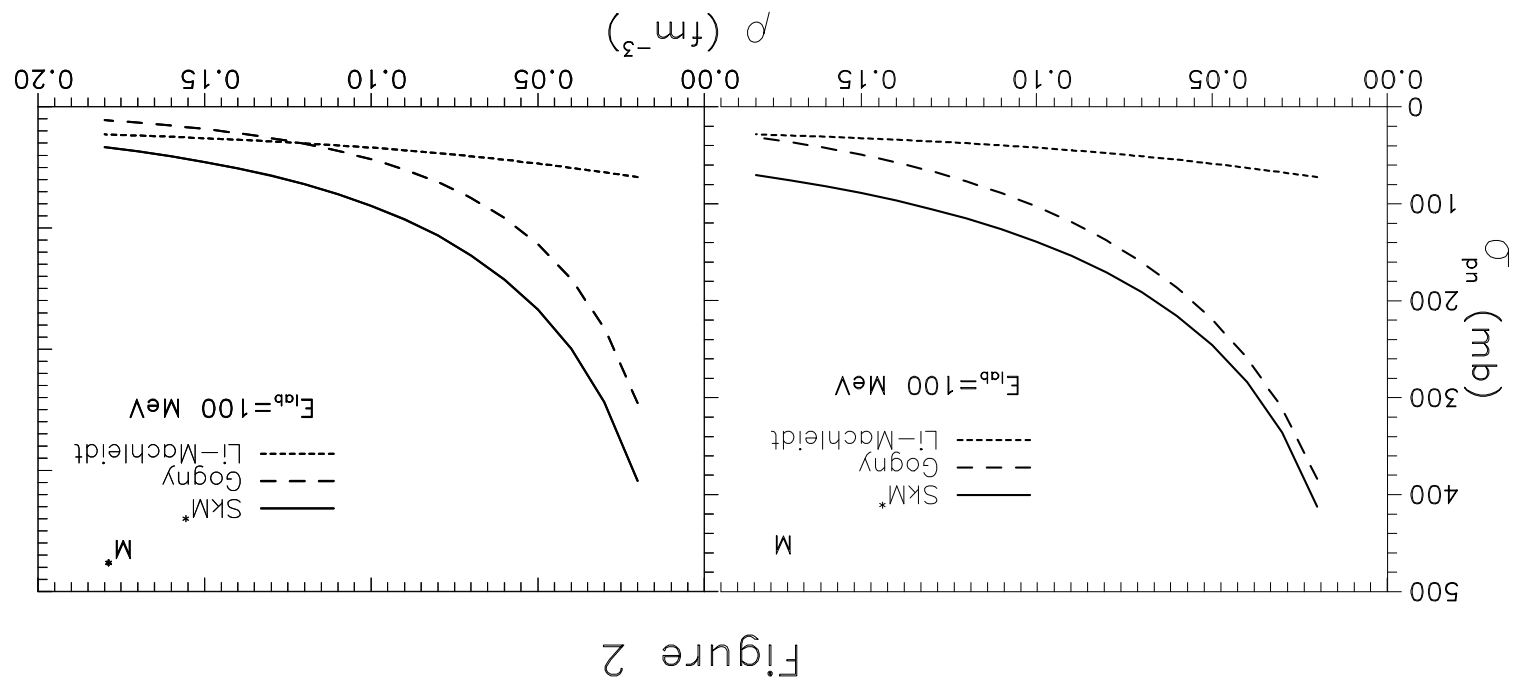




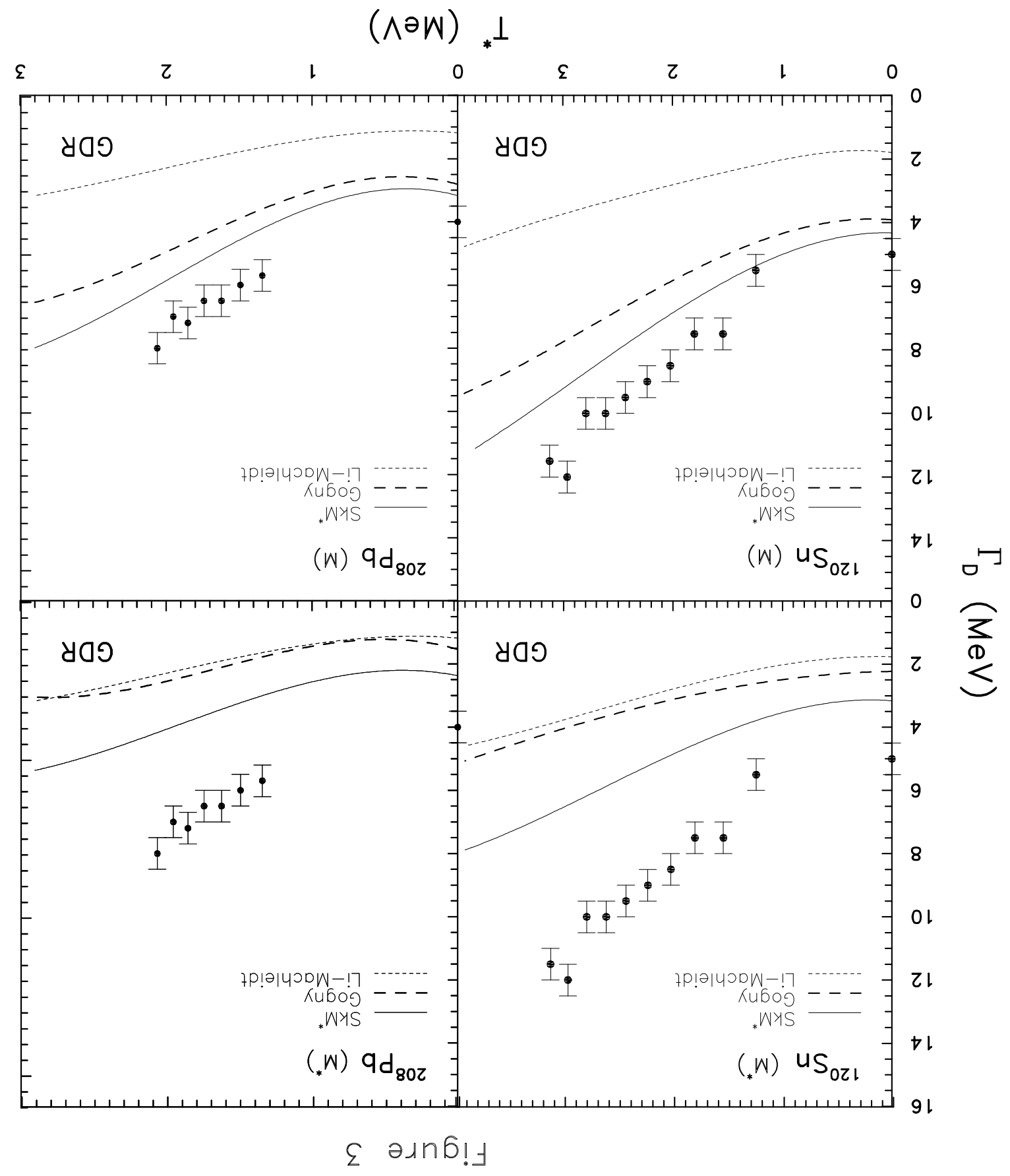




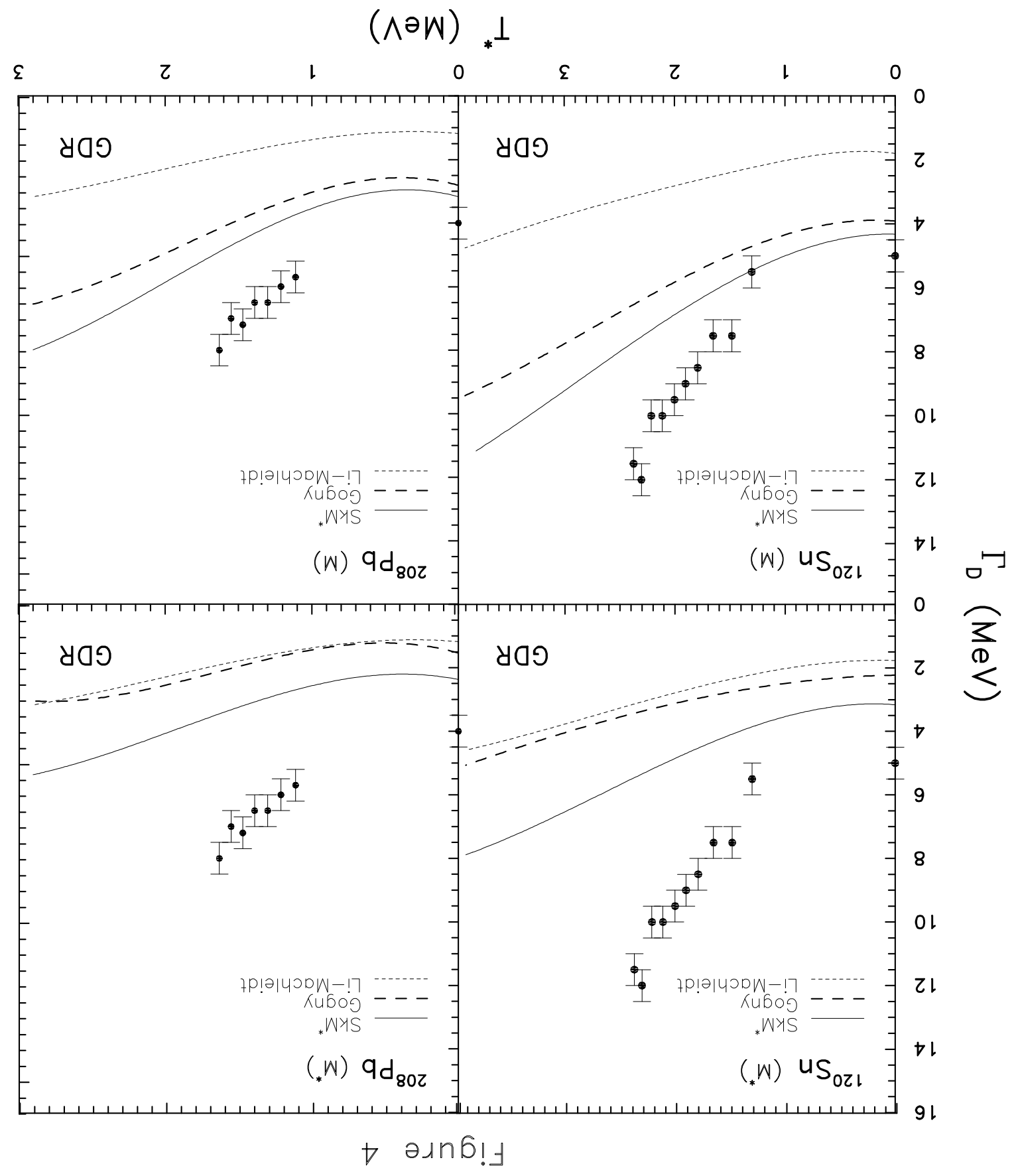

